\title{
FIXED POINT THEOREMS FOR MAPPINGS OF NONEXPANSIVE TYPE
}

\author{
TECK-CHEONG LIM
}

\begin{abstract}
Constructive fixed point theorems for single-valued and compact-valued nonexpansive mappings, which map a closed convex subset $C$ of a Banach space $X$ into $X$ and send the boundary of $C$ relative to $X$ into $C$, are given. Mappings for which the method of asymptotic center applies are also considered.
\end{abstract}

In this note we consider fixed point theorems for mappings $f: C \rightarrow X$ of the following types:

(1) $f$ is single-valued, nonexpansive and $f(\partial C) \subset C$.

(2) $f$ is multivalued, nonexpansive and $f(x) \subset C$ for $x \in \partial C$.

(3) $f$ is single-valued and satisfies the condition

$$
\lim _{m} \sup \limsup _{n}\left\|f^{m}(x)-f^{n}(y)\right\| \leqslant k \lim \sup _{n}\left\|x-f^{n}(y)\right\|
$$

for some $k>0$. In the sequel, unless otherwise stated, $X$ denotes a uniformly convex Banach space, $C$ a closed convex nonempty bounded subset of $X$ and $\partial C$ the boundary of $C$ relative to $X$. If $f: C \rightarrow X$ is a mapping such that $f(\partial C) \subset C$, we define $F: C \rightarrow C$ by putting

$$
\begin{aligned}
F(x) & =f(x) \quad \text { if } f(x) \in C, \\
& =\text { the point where the line segment }[x, f(x)] \text { and } \partial C \text { intersect } \\
& \text { if } f(x) \notin C .
\end{aligned}
$$

If $f$ is a contraction, it is known that $f$ has a fixed point (Assad and Kirk [1]). If $f$ is nonexpansive, by considering the contractions

$$
f_{\lambda}(x)=\lambda x_{0}+(1-\lambda) f(x), \quad 0<\lambda<1, x_{0} \in C,
$$

we get for each $\lambda$, a fixed point $x_{\lambda}$ of $f_{\lambda}$, and it follows that $\left\|x_{\lambda}-f\left(x_{\lambda}\right)\right\| \rightarrow 0$ as $\lambda \rightarrow 0$.

The asymptotic center w.r.t. $C$ of a bounded sequence $\left\{x_{n}\right\}$ in $X$ is the unique point in $C$ at which the function

$$
r(x)=\lim _{n} \sup _{n}\left\|x-x_{n}\right\|, \quad x \in C,
$$

attains its minimum (Edelstein [2]). $r(x)$ is clearly a convex function. We begin with the following

Received by the editors November 5, 1976.

AMS (MOS) subject classifications (1970). Primary 47H10, 46B99. 
Proposition 1. Suppose that the asymptotic center $c$ w.r.t. $C$ of $\left\{x_{n}\right\}$ is an interior point of $C$. Then $c$ is also the asymptotic center of $\left\{x_{n}\right\}$ w.r.t. X.

Proof. If not, let $c^{\prime}$ be the asymptotic center of $\left\{x_{n}\right\}$ w.r.t. $X$. Since $c$ is an interior point of $C$, the line segment $\left[c, c^{\prime}\right]$ has a nontrivial intersection with $C$, i.e. there exists $0<\lambda<1$ such that $c_{1}=\lambda c+(1-\lambda) c^{\prime} \in C$. Since $r\left(c^{\prime}\right)$ $\leqslant r(c)$, it follows from the convexity of $r(x)$ that $r\left(c_{1}\right) \leqslant r(c)$. This contradicts the uniqueness of the asymptotic center.

For nonexpansive mappings existence of fixed points of $f$ was proved by Kirk [6] under a more general setting. In case $f: C \rightarrow C$, part (a) of the following theorem was proved by Edelstein [2].

THEOREM 1. Let $f: C \rightarrow X$ be a nonexpansive mapping with $f(\partial C) \subset C$.

(a) The asymptotic center of the sequence $\left\{x, F(x), F^{2}(x), \ldots\right\}$ w.r.t. $C$ is a fixed point of $f$.

(b) If $x_{n}-f\left(x_{n}\right) \rightarrow 0$, then the asymptotic center of $\left\{x_{n}\right\}$ w.r.t. $C$ is a fixed point of $f$.

Proof. Let $c$ be the asymptotic center of $\left\{x, F(x), F^{2}(x), \ldots\right\}$ w.r.t. $C$. For each $n$, consider $\left\|f(c)-F^{n}(x)\right\|$.

Case (i). $f\left(F^{n-1}(x)\right) \in C$. Then $F^{n}(x)=f\left(F^{n-1}(x)\right)$ and

$$
\left\|f(c)-F^{n}(x)\right\| \leqslant\left\|c-F^{n-1}(x)\right\| .
$$

Case (ii). $f\left(F^{n-1}(x)\right) \notin C$. Then $F^{n-1}(x)=f\left(F^{n-2}(x)\right)$ for, otherwise, $F^{n-1}(x) \in \partial C$ and hence $f\left(F^{n-1}(x)\right) \in C$ by hypothesis, a contradiction. Thus

$$
\begin{aligned}
F^{n}(x) & =\lambda F^{n-1}(x)+(1-\lambda) f\left(F^{n-1}(x)\right) \\
& =\lambda f\left(F^{n-2}(x)\right)+(1-\lambda) f\left(F^{n-1}(x)\right)
\end{aligned}
$$

and

$$
\left\|f(c)-F^{n}(x)\right\| \leqslant \lambda\left\|F^{n-2}(x)-c\right\|+(1-\lambda)\left\|F^{n-1}(x)-c\right\| .
$$

It follows from (1) and (2) that

$$
\lim _{n} \sup \left\|f(c)-F^{n}(x)\right\| \leqslant \lim _{n} \sup \left\|c-F^{n}(x)\right\| .
$$

If $f(c) \in C$, then $f(c)=c$ by the uniqueness of asymptotic center. Otherwise $f(c) \notin C$ and $c$ must be an interior point of $C$. By Proposition $1, c$ is also the asymptotic center of $\left\{F^{n}(x)\right\}$ w.r.t. $X$, and hence by (1) and the uniqueness, $f(c)=c$, contradicting $f(c) \notin C$. Hence $f(c) \in C$ and $f(c)=c$.

For part (b), let $c$ be the asymptotic center of $\left\{x_{n}\right\}$ w.r.t. $C$. Then

$$
\begin{aligned}
\left\|f(c)-x_{n}\right\| & \leqslant\left\|f(c)-f\left(x_{n}\right)\right\|+\left\|f\left(x_{n}\right)-x_{n}\right\| \\
& \leqslant\left\|c-x_{n}\right\|+\left\|f\left(x_{n}\right)-x_{n}\right\| .
\end{aligned}
$$

Taking lim sup we have, lim sup $\left\|f(c)-x_{n}\right\| \leqslant \lim \sup \left\|c-x_{n}\right\|$.

The following theorem slightly generalizes Theorem 1 in [9].

TheOREM 2. Denote by $\mathcal{C}(X)$ the family of compact nonempty subsets of $X$ 
equipped with the Hausdorff metric. If $f: C \rightarrow \mathcal{C}(X)$ is nonexpansive and $f(x) \subset C$ for all $x \in \partial C$, then $f$ has a fixed point.

Proof. As in [9], there exist sequences $\left\{x_{n}\right\} \subset C$ and $\left\{y_{n}\right\} \subset X$ such that $y_{n} \in f\left(x_{n}\right),\left\|x_{n}-y_{n}\right\| \rightarrow 0$ and all subsequences of $\left\{x_{n}\right\}$ have the same asymptotic center w.r.t $C$. Arguing as in [9], there exists $p \in f(c)$ such that $\lim \sup \left\|p-x_{n}\right\| \leqslant \lim \sup \left\|c-x_{n}\right\|$. If $f(c) \subset C$, then $p \in C$ and $p=c$ by the uniqueness of asymptotic center w.r.t. $C$; if not, $c$ must be an interior point and, by Proposition $1, p=c$. Hence $c \in f(c)$.

THEOREM 3. Let $C$ be a subset of a Banach space such that every bounded net in $C$ has an asymptotic center, e.g. a closed convex subset of a reflexive Banach space. Let $\Omega$ be the first uncountable ordinal and $x_{\alpha}, \alpha<\Omega$, be a transfinite sequence in $C$ indexed by ordinals less than $\Omega$ such that $x_{\gamma}$ is an asymptotic center [8] of $x_{\eta}, \eta<\gamma$, whenever $\gamma$ is a limit ordinal. Define

$$
\begin{aligned}
& R_{1}\left(x_{\gamma}\right)=\sup \left\{\left\|x_{\gamma}-x_{\gamma+n}\right\|: n=1,2, \ldots\right\}, \\
& R_{2}\left(x_{\gamma}\right)=\lim \sup \left\{\left\|x_{\gamma}-x_{\gamma+n}\right\|: n=1,2, \ldots\right\}, \\
& R_{3}\left(x_{\gamma}\right)=\inf _{x \in C} \lim \sup \left\{\left\|x_{\gamma}-x_{\gamma+n}\right\|: n=1,2, \ldots\right\}
\end{aligned}
$$

and suppose $f: C \rightarrow C$ is a function such that $f\left(x_{\gamma}\right)=x_{\gamma+1}$. If the following condition is satisfied for some $i=1,2,3$ :

$$
R_{i}\left(x_{\delta}\right) \leqslant R_{i}\left(x_{\gamma}\right), \quad \delta>\gamma \text { and } R_{i}\left(x_{\gamma}\right)>R_{i}\left(x_{\gamma+\omega}\right) \quad \text { if } R_{i}\left(x_{\gamma}\right)>0 \text {, }
$$

then (i) $f$ has a fixed point if $i=1$, (ii) there exists a point $x$ such that $\lim _{n} f^{n}(x)=x$ if $i=2$, and (iii) there exists a point $x$ such that $\lim _{n} f^{n}(x)$ exists if $i=3$.

PROof. The transfinite sequence of nonnegative real numbers $R_{i}\left(x_{\gamma}\right), \gamma<\Omega$ is decreasing and must be eventually constant. There exists a point $x_{\gamma}$ such that $R_{i}\left(x_{\gamma}\right)=R_{i}\left(x_{\gamma+\omega}\right)$ and hence $R_{i}\left(x_{\gamma}\right)=0$. The conclusion in the theorem then follow immediately.

The proofs of the following lemmas were given in [10].

Lemma 1. Let $\left\{x_{n}\right\}$ be a bounded sequence in $X$. For each $x \in X$ let $r(x)=\lim \sup _{n}\left\|x-x_{n}\right\|$. Let $\delta(\varepsilon)$ be the modulus of convexity of $X$.

(i) If $r(x) \leqslant d, r(y) \leqslant d$ and $\|x-y\| \geqslant \varepsilon$, then $r((x+y) / 2) \leqslant d(1-$ $\delta(\varepsilon / d))($ or $\delta(\varepsilon / d-)$ if $\varepsilon / d=2)$.

(ii) $|r(x)-r(y)| \leqslant\|x-y\| \leqslant r(x)+r(y)$.

Lemma 2. Suppose that in Lemma $1,\left\{x_{n}\right\}$ is relatively compact and $C$ is a closed convex subset of $X$. Then the asymptotic center of $\left\{x_{n}\right\}$ w.r.t. $C$ is the Chebyshev center of the set of subsequential limits of $\left\{x_{n}\right\}$ w.r.t. C.

The following two theorems are slight generalizations and modifications of Theorems 1 and 2 in Kirk [7] and Theorem 1 in Goebel and Kirk [4]. The first illustrates the method used in Theorem 3 with $R_{3}\left(x_{\omega^{2}}\right)=0$. Let $X$ be a 
Banach space with $\delta(1)>0 . X$ is reflexive (James [5]). Let $\alpha>1$ be the solution of $\alpha(1-\delta(1 / \alpha))=1$.

TheOREM 4. Let $X$ be defined as above and $C$ a closed convex subset of $X$. Let $f: C \rightarrow C$ be a mapping satisfying the following condition:

(i) $\quad \lim \sup \lim \sup \left\|f^{m}(x)-f^{n}(y)\right\| \leqslant k \lim \sup \left\|x-f^{n}(y)\right\|$, where $0<k<\alpha$. Then there exists an $x$ with $\lim _{n} f^{n}(x)=x$. If $f^{N}$ is continuous for some $N>0$, then $x$ is a fixed point of $f$.

Proof. We can assume $1<k<\alpha$. Choose $k^{\prime}, k<k^{\prime}<\alpha$. Define $x_{\gamma}$ for $r<\omega^{2}$ as in Theorem 3. Denote $y_{n}=x_{n \omega}$. For each $n$, let

$$
r_{n}(x)=\lim _{m} \sup \left\|x-f^{m}\left(y_{n}\right)\right\| .
$$

Thus $f_{n-1}\left(y_{n}\right)=R_{3}\left(y_{n-1}\right)$ in the notation of Theorem 3. We shall write $s_{n}$ for $r_{n-1}\left(y_{n}\right)$.

If $s_{n}=0$ for some $n$, then $f^{i}\left(y_{n-1}\right) \rightarrow y_{n}$ and it follows from (i) that $f^{i}\left(y_{n}\right) \rightarrow y_{n}$. Therefore we assume $s_{n}>0$.

Claim.

$$
r_{n}\left(y_{n}\right) \leqslant\left(k^{\prime} / \alpha\right) r_{n-1}\left(y_{n-1}\right) .
$$

Since $k^{\prime}>1$, we have $r_{n-1}\left(y_{n}\right) \leqslant k^{\prime} s_{n}$. For sufficiently large $m$, we have, from (i), $r_{n-1}\left(f^{m}\left(y_{n}\right)\right) \leqslant k^{\prime} s_{n}$. If $\left\|y_{n}-f^{m}\left(y_{n}\right)\right\|>\left(k^{\prime} / \alpha\right) s_{n}$, then by Lemma 1

$$
r_{n-1}\left(\frac{y_{n}+f^{m}\left(y_{n}\right)}{2}\right) \leqslant k^{\prime} s_{n}\left(1-\delta\left(\frac{1}{\alpha}\right)\right)=\frac{k^{\prime}}{\alpha} s_{n}<s_{n}=R_{3}\left(y_{n-1}\right),
$$

a contradiction to the definition of $y_{n}$. Hence we have $\left\|y_{n}-f^{m}\left(y_{n}\right)\right\| \leqslant$ $\left(k^{\prime} / \alpha\right) s_{n}$ for sufficiently large $m$, which implies

$$
r_{n}\left(y_{n}\right) \leqslant\left(k^{\prime} / \alpha\right) s_{n} \leqslant\left(k^{\prime} / \alpha\right) r_{n-1}\left(y_{n-1}\right) \text {. }
$$

From (ii) it follows that $r_{n}\left(y_{n}\right) \leqslant\left(k^{\prime} / \alpha\right)^{n-1} r_{1}\left(y_{1}\right)$.

Since

$$
\begin{aligned}
\left\|y_{n+1}-y_{n}\right\| & \leqslant r_{n+1}\left(y_{n+1}\right)+r_{n+1}\left(y_{n}\right) \leqslant 2 r_{n+1}\left(y_{n+1}\right) \\
& \leqslant 2\left(k^{\prime} / \alpha\right)^{n} r_{1}\left(y_{1}\right),
\end{aligned}
$$

the sequence $y_{n}$ is a Cauchy sequence. Let $z$ denote its limit. It follows from the inequalities (from Lemma 1)

$r_{n}(z) \leqslant r_{n}\left(y_{n}\right)+\left\|y_{n}-z\right\|$,

$\left\|f^{i}(z)-z\right\| \leqslant r_{n}\left(f^{i}(z)\right)+r_{n}(z)$, and

$\lim \sup _{i} r_{n}\left(f^{i}(z)\right) \leqslant k r_{n}(z)$ (from (i)) that $r_{n}(z) \rightarrow 0$ and $f^{i}(z) \rightarrow z$.

If $f^{N}$ is continuous for some $N$, then $f(z)=\lim _{p} f^{N p+1}(z)=z$.

THEOREM 5. Let $K$ be a nonempty compact convex subset of a Banach space $X$. Let $f: K \rightarrow K$ be a mapping satisfying

$$
\lim _{m} \sup \lim \sup _{n}\left\|f^{m}(x)-f^{n}(y)\right\| \leqslant \lim \sup _{n}\left\|x-f^{n}(y)\right\| .
$$


Then there exists a point $x$ such that $\lim _{n} f^{n}(x)=x$. If $f^{N}$ is continuous for some $N>0$, then $f(x)=x$.

Proof. Let $M$ be a subset of $K$ minimal w.r.t. being nonempty, closed, convex and that for every $x \in M$, every subsequential limit of $\left\{f^{n}(x)\right\}$ is in $M$. Such an $M$ exists by a standard argument of Zorn's Lemma. Suppose that $M$ consists of more than one point. Let $x_{0} \in M$ and let $C$ be the asymptotic center of $\left\{f^{n}\left(x_{0}\right)\right\}$ w.r.t. $M$. By Lemma 2, $C$ is the Chebyshev center w.r.t. $M$ of the set $D$ of subsequential limits of $\left\{f^{n}\left(x_{0}\right)\right\}$ in $M$. By hypothesis $D \subset M$. Since $M$ has normal structure [11], $C$ is a proper subset of $M$. For each $x \in K$, let $r(x)=\lim \sup _{n}\left\|x-f^{n}\left(x_{0}\right)\right\|$ and $r_{1}=\inf \{r(x): x \in M\}$. Then $C=\left\{x \in M: r(x)=r_{1}\right\}$. If $y \in C$, then it follows from condition (A) that $\lim \sup _{i} r\left(f^{i}(y)\right) \leqslant r(y)$. Let $\left\{f^{n_{i}}(y)\right\}$ be a convergent subsequence of $\left\{f^{n}(y)\right\}$ with limit $z$. By continuity of $r$, we have

$$
\limsup _{i} r\left(f^{n_{i}}(y)\right)=r(z) \leqslant \limsup _{n} r\left(f^{n}(y)\right) \leqslant r(y)=r_{1} .
$$

Since $z \in M$ we must have $r(z)=r_{1}$, i.e. $z \in C$. This shows that $C$ is a nonempty closed convex proper subset of $M$ and every subsequential limit of $\left\{f^{n}(x)\right\}$ is in $C$ for every $x \in C$, a contradiction to the minimality of $M$. Hence $M$ consists of one point $z_{0}$. Obviously we have $\lim f^{n}\left(z_{0}\right)=z_{0}$. The last part of the theorem follows from the proof of Theorem 4.

Finally we remark that inequality (i) in Theorem 4 is implied by each of the following:

$$
\begin{aligned}
& \left\|f^{i}(x)-f^{i}(y)\right\| \leqslant k_{i}\|x-y\|, \quad i \geqslant N, \quad k_{i} \rightarrow 1 \quad[3] \\
& \left\|f^{i}(x)-f^{i}(y)\right\| \leqslant k\|x-y\|, \quad i \geqslant N \quad[4] \\
& \lim \sup \sup _{y \in C}\left\{\left\|f^{i}(x)-f^{i}(y)\right\|-\|x-y\|\right\} \leqslant 0 \quad[7]
\end{aligned}
$$

\section{REFERENCES}

1. N. A. Assad and W. A Kirk, Fixed point theorems for set-valued mappings of contractive type, Pacific J. Math. 43 (1972), 553-562.

2. M. Edelstein, Fixed point theorems in uniformly convex Banach spaces, Proc. Amer. Math. Soc. 44 (1974), 369-374.

3. K. Goebel and W. A Kirk, A fixed point theorem for asymptotically nonexpansive mappings, Proc. Amer. Math. Soc. 30 (1972), 171-174.

4. __ A fixed point theorem for transformations whose iterates have uniform Lipschitz constant, Studia Math. 47 (1973), 135-140.

5. R. C. James, Uniformly non-square Banach spaces, Ann. of Math. (2) 80 (1964), 542-550.

6. W. A. Kirk, Fixed point theorem for nonlinear nonexpansive and generalized contraction mappings, Pacific J. Math. 38 (1971), 89-94.

7. Fixed point theorems for non-Lipschitzian mappings of asymptotically nonexpansive type, Israel J. Math. 17 (1974), 339-346.

8. T.-C. Lim, Characterization of normal structure, Proc. Amer. Math. Soc. 43 (1974), 313-319. 
9. Banach space, Bull. Amer. Math. Soc. 80 (1974), 1123-1126.

10. The asymptotic center and fixed point theorems for nonexpansive mappings, $\mathrm{Ph} . \mathrm{D}$. Thesis, Dalhousie Univ., 1974.

11. Z. Opial, Lecture notes on nonexpansive and monotone mappings in Banach spaces, Center for Dynamical Systems, Brown Univ., Providence, R. I., 1967.

Lee Kong Chian Institute of Mathematics and Computer Science, Nanyang University, SINGAPORE 22 